\title{
Does Twitter language reliably predict heart disease? A commentary on Eichstaedt et al. (2015a)
}

\author{
Nicholas J L Brown ${ }^{\text {Corresp., }}{ }^{1}$, James C Coyne ${ }^{1}$ \\ ${ }^{1}$ University Medical Center, University of Groningen, Groningen, Netherlands \\ Corresponding Author: Nicholas J L Brown \\ Email address: nicholasjlbrown@gmail.com
}

We comment on Eichstaedt et al.'s (2015a) claim to have shown that language patterns among Twitter users, aggregated at the level of U.S. counties, predicted county-level mortality rates from atherosclerotic heart disease (AHD), with "negative" language being associated with higher rates of death from AHD and "positive" language associated with lower rates. First, we examine some of Eichstaedt et al.'s apparent assumptions about the nature of AHD, as well as some issues related to the secondary analysis of online data and to considering counties as communities. Next, using the data files supplied by Eichstaedt et al., we reproduce their regression- and correlation-based models, substituting mortality from an alternative cause of death-namely, suicide-as the outcome variable, and observe that the purported associations between "negative" and "positive" language and mortality are reversed when suicide is used as the outcome variable. We identify numerous other conceptual and methodological limitations that call into question the robustness and generalizability of Eichstaedt et al.'s claims, even when these are based on the results of their ridge regression/machine learning model. We conclude that there is no good evidence that analyzing Twitter data in bulk in this way can add anything useful to our ability to understand geographical variation in AHD mortality rates. 
1

2

3

4

5

6

7

8

9 Acknowledgements

10 We thank Casper Albers, Daniël Lakens, and a number of colleagues who wished to remain

11 anonymous for helpful discussions during the writing of this article. All errors and omissions

12 remain the responsibility of the authors alone.

\section{Does Twitter Language Reliably Predict Heart Disease?}

\section{A Commentary on Eichstaedt et al. (2015a)}

\author{
Nicholas J. L. Brown \\ James C. Coyne \\ University of Groningen
}

13 


\section{Abstract}

16 We comment on Eichstaedt et al.'s (2015a) claim to have shown that language patterns among

17 Twitter users, aggregated at the level of U.S. counties, predicted county-level mortality rates

18 from atherosclerotic heart disease (AHD), with "negative" language being associated with higher

19 rates of death from AHD and "positive" language associated with lower rates. First, we examine

20 some of Eichstaedt et al.'s apparent assumptions about the nature of AHD, as well as some issues

21 related to the secondary analysis of online data and to considering counties as communities.

22 Next, using the data files supplied by Eichstaedt et al., we reproduce their regression- and

23 correlation-based models, substituting mortality from an alternative cause of death - namely,

24 suicide - as the outcome variable, and observe that the purported associations between

25 "negative" and "positive" language and mortality are reversed when suicide is used as the

26 outcome variable. We identify numerous other conceptual and methodological limitations that

27 call into question the robustness and generalizability of Eichstaedt et al.'s claims, even when

28 these are based on the results of their ridge regression/machine learning model. We conclude that

29 there is no good evidence that analyzing Twitter data in bulk in this way can add anything useful

30 to our ability to understand geographical variation in AHD mortality rates. 
33 Twitter users, aggregated at the level of U.S. counties, were predictive of mortality rates from

34 atherosclerotic heart disease (AHD) in those counties, with "negative" language (expressing

35 themes such as disengagement or negative relationships) being associated with higher rates of

36 death from AHD and "positive" language (e.g., upbeat descriptions of social interactions or

37 positive emotions) being associated with lower AHD mortality. Eichstaedt et al. examined a

38 variety of measures to demonstrate the associations between Twitter language patterns and AHD,

39 including (a) the frequency of usage of individual words associated with either positive or

40 negative feelings or behaviors, (b) the tendency of Twitter users to discuss "positive" (e.g.,

41 skilled occupations) or "negative" (e.g., feelings of boredom) topics, and (c) an omnibus model

42 incorporating all of their Twitter data, whose performance they compared with one using only

43 "traditional" predictors such as health indicators and demographic and socioeconomic variables.

44 The claims made by Eichstaedt et al. (2015a) attracted considerable attention in the

45 popular media (e.g., Izadi, 2015; Jacobs, 2015; Singal, 2015), with many of these articles being

46 based in large part on the Association for Psychological Science's (2015) own press release.

47 However, a close examination of Eichstaedt et al.'s article and data appears to reveal a number of potential sources of distortion and bias in its assumptions about the nature of AHD, the use of

49 Twitter data as a proxy for the socioemotional environment and people's health, and the use of counties as the unit of analysis. Some of these problems are immediately obvious from reading

51 Eichstaedt et al.'s article, while others only manifested themselves in the testing of the relevant 52 data that we undertook.

Here, we first present a selection of the main problems that we identified when reading

54 Eichstaedt et al.'s (2015a) article. We then expose further problems that emerged after we 
55 scrutinized the original data and also cross-validated the application of Eichstaedt et al.'s models

56 to a different mortality outcome variable, namely suicide. We end with a discussion of some

57 broader implications for the use of large-scale sources of data to draw conclusions about health

58 and human behavior based on sophisticated computer models.

59 Issues related to the idea of psychological causes of AHD

60 Perhaps a large part of the appeal of Eichstaedt et al.'s (2015a) claims about the potential

61 for community-level psychological factors - notably, those that purportedly lead Twitter users to

62 either make aggressive or otherwise anti-social outbursts, or, conversely, express upbeat and

63 positive sentiments - to somehow affect local rates of death from AHD is that these claims echo

64 the common belief, repeated in the first sentence of Eichstaedt et al.'s abstract, that hostile or

65 highly stressed individuals are more susceptible to cardiovascular problems. The notion that a

66 loudmouthed, dominating, aggressive person is somehow more likely to suddenly drop dead

67 from a heart attack is widespread in lay or pop psychology thinking (e.g., Riggio, 2014), perhaps

68 at least in part because it provides some comfort to people on the receiving end of such

69 individuals' behavior. Indeed, although Eichstaedt et al. did not use the term "Type A

70 personality" in their article, this stereotype - characterized by a tendency towards aggression and

71 a variety of other negative interpersonal characteristics, as well as greater susceptibility to

72 cardiovascular problems - is a staple part of the popular culture surrounding the relation between

73 mental and physical health (e.g., Wilson, 2009). Yet, despite initial promising findings

74 suggesting a link between Type A behavior pattern (TABP) and cardiac events and mortality in

75 small samples (Friedman \& Rosenman, 1959), an accumulation of evidence from more recent

76 large-scale studies has consistently failed to show reliable evidence for such an association

77 (Kuper, Marmot, \& Hemingway, 2002). Appearances of positive findings could be generated 
using a range of distress or negative affectivity variables (Smith, 2006). However, it was then recognized that negative affectivity could not readily be separated from a full range of antecedent and concurrent biological, psychological, and social factors. At best, negative affectivity is likely to be no more than a non-informative risk marker (Ormel et al., 2004), not a risk factor for AHD. Its apparent predictive power is greatly diminished with better specification and measurement of confounds (Smith, 2011).

A recent large scale study of over 150,000 Australians (Welsh et al, 2017) provides a typical example of the problem. Significant associations between levels of psychological distress and incidence of ischemic heart disease (a superordinate category in the ICD-10 classification that includes AHD as a subcategory) decreased as adjustments were made for demographic and behavioral characteristics until measurement error and residual confounding seemed to account for any remaining association. As the authors put it, "A substantial part of the distress-IHD association is explained by confounding and functional limitations .... Emphasis should be on psychological distress as a marker of healthcare need and IHD risk, rather than a causative factor" (Welsh et al., 2017, p. 1).

In contrast to $\mathrm{TABP}$, socioeconomic conditions have long been identified as playing a role in the development of AHD. For example, Clark, DesMeules, Luo, Duncan, and Wielgosz (2009) noted the importance of access to good-quality healthcare and early-life factors such as parental socioeconomic status. However, no county-level measure of either of those variables appeared in Eichstaedt et al.’s (2015a) model or data set.

\section{Issues related to the etiology of AHD}

As the single most common ICD-10 cause of mortality in the United States, AHD might have seemed like a natural choice of outcome variable for a study such as that of Eichstaedt et al. 
101 (2015a). However, it is important to take into account some aspects of the nature and course of

102 AHD. It is a chronic disease that typically develops asymptomatically over decades. The first

103 recognition of AHD often follows an event such as an acute myocardial infarction or some other

104 sudden incident, reflecting the fact that the cumulative build-up of plaque over time has finally

105 caused a blockage of the arteries (Funk, Yurdagul, \& Orr, 2012) rather than any specific change

106 in the immediately preceding time period. Indeed, disagreement among physicians as to whether

107 the cause of death is AHD or some other cardiac event is common (Mant et al., 2006). A

108 definitive post-mortem diagnosis of AHD may require an autopsy, yet the number of such

109 procedures performed in the United States has halved in the past four decades (Hoyert, 2011).

110 In contrast to AHD, there is another cause of death for which the existence of an

111 association with the victim's recent psychological state is widely accepted, namely suicide

112 (Franklin et al., 2017; O'Connor \& Nock, 2014). Although suicide can be the result of long-term

113 mental health problems and other stressors, a person's psychological state in the months and

114 days leading up to the point at which they take their own life clearly has a substantial degree of

115 relevance to their decision. Hence, we might expect any county-level psychological factors that

116 act directly on the health and welfare of members of the local community to be more closely

117 reflected in the mortality statistics for suicide than those for a chronic disease such as AHD.

118 Issues related to the secondary analysis of data collected online

119 In the introduction to their article, Eichstaedt et al. (2015a) invoked Google's use of

120 search queries related to influenza, "providing earlier indication of disease spread than the

121 Centers for Disease Control and Prevention" (p. 160) as a (presumably positive) example of how

122 "digital epidemiology can support faster response and deeper understanding of public-health

123 threats than can traditional methods" (p. 160). However, both Google's project to infer a relation 
124 between searches for certain terms and the immediate prevalence of an acute infectious disease,

125 and Eichstaedt et al.'s attempt to correlate certain kinds of communication with chronic

126 cardiovascular conditions, suffer from the fundamental problem that they are attempting to

127 extract some kind of signal from what may very well be a large amount of noise. In fact, before it

128 was quietly shut down in August 2015, Google Flu Trends (GFT) failed—in some cases

129 spectacularly - to correctly predict the seasonal spread of influenza in the United States on

130 several occasions. As Lazer, Kennedy, King, and Vespignani (2014) put it, in their review of the

131 problems that beset GFT, "The core challenge is that most big data that have received popular

132 attention are not the output of instruments designed to produce valid and reliable data amenable

133 for scientific analysis" (p. 1203) — a remark that clearly also applies to the notionally random

134 selection of tweets that constitute the Twitter "Garden Hose" data, especially since, as we show

135 below, this selection may not have been entirely random.

136 As well as the limitations noted in Eichstaedt et al.'s article (2015a) and Supplemental

137 Material (2015b) — such as the difference in Twitter user density across counties, and the fact

138 that $7 \%$ of tweets were mapped to the wrong county — we note that there is also a potential

139 source of bias in the geographical coding of their Twitter data, namely the assumption that the

140 users who provided enough information to allow their tweets to be associated with a county

141 represented an unbiased sample of Twitter users in that county. This requires people of all ages,

142 genders, socio-economic status levels, and ethnic backgrounds to be equally likely to either list

143 their city and state in their profile, or to enable geotagging of their Tweets. However, it seems

144 entirely plausible that certain categories of individuals might be more likely to self-censor their

145 profile information than others (for example, it could be that people who do not wish to reveal 
146 their location are more or less restrained in their use of hostile language). Given that only $16 \%$ of

147 tweets could be mapped to counties, any bias in this area could have substantial consequences.

148 Issues associated with considering counties as communities

149 As Eichstaedt et al. (2015a, p. 160) themselves noted, "Counties are the smallest

150 socioecological level for which most CDC health variables and U.S. Census information are

151 available." Thus, these authors seem to have acknowledged that their use of counties as their unit

152 of analysis was driven principally by (eminently reasonable) practical constraints. However,

153 Eichstaedt et al.'s subsequent interpretation of their results (e.g., "language expressed on Twitter

154 revealed several community-level psychological characteristics that were significantly associated

155 with heart-disease mortality risk," p. 164) requires that counties also constitute meaningful

156 communities. Indeed, this interpretation also implies that any psychological mechanism that

157 might account for the relationship between the vocabulary of Twitter users and the health

158 outcomes of the wider population within any given county works in a similar way across all

159 counties. Yet there seem to be several reasons to question such assumptions. First, the size and

160 population of U.S. counties varies widely; both their land areas (ranging from 1.999 to

$161144,504.789$ square miles), and their 2010 populations (from 82 to 9,818,605) span five orders of

162 magnitude. Second, across the United States, the political and economic importance of counties

163 as a level of government (between the municipality and state level) is highly variable, being

164 generally greater in the South (Benton, 2002, p. 7; Haider-Merkel, 2009, p. 723); indeed, in

165 Connecticut, Rhode Island, and much of Massachusetts, counties have no governmental function

166 whatsoever. Third, it is not clear that many Americans identify at all closely with their county as

167 a source of community (Kilgore, 2012). Fourth, within counties, socioeconomic and other factors

168 often vary enormously: 
[I]n New York County, New York, ... neighborhoods range from the Upper East Side and SoHo to Harlem and Washington Heights. ... [I]n San Mateo County, California, ... neighborhoods range from the Woodside estates of Silicon Valley billionaires to the Redwood City bungalows of Mexican immigrants. (Abrams and Fiorina, 2012, p. 206) difficult to conceive of a county-level factor, or set of factors, that might be associated with both Twitter language and AHD prevalence with any degree of consistency across the United States.

Eichstaedt et al. (2015a, p. 166) cited two meta-analyses (Leyland, 2005; Riva, Gauvin, \&

Barnett, 2007), which they claimed provided support for the idea that "the aggregated outcomes," but both of those meta-analyses were based predominantly on small and relatively homogeneously-sized geographical areas (and Leyland's study examined only health-related behaviors, not outcomes). The approximate population of each area in Leyland's study was 5,000, while Riva et al. reported a median area population of 8,600; compare these with Eichstaedt et al.'s mean county population of 206,000 and median of 78,000. As Beyer, Schultz, and Rushton (2008, p. 40) put it, "The county often represents an area too large to use in determining true, local patterns of disease." also raises the question of whether specific data items can be aggregated in a meaningful way to create a collective "characteristic" (cf. Subramanian, Jones, Kaddour, \& Krieger, 2008). For example, it is difficult to know what interpretation to place on the median individual household income of a county, especially one with a highly diverse population. It is also worth noting that,

191 as reported by Eichstaedt et al. in their Supplemental Tables document (2015c), the "county- 
192 level" data for all of the variables that measure "county-level" health in their study (obesity,

193 hypertension, diabetes, and smoking) are in fact statistical estimates derived from state-level data

194 using "Bayesian multilevel modeling, multilevel logistic regression models, and a Markov Chain

195 Monte Carlo simulation method" (p. DS7). However, Eichstaedt et al. provided no estimates of

196 the possible errors or biases that the use of such techniques might introduce.

197 Based on the above observations, we decided to reproduce a number of Eichstaedt et al.'s

198 (2015a) analyses, using their original data files in conjunction with other sources of information,

199 to see whether the assumptions made by these authors stand up to a critical examination.

200

201

202

203

204

205

206

\section{Method}

We were able to download all of Eichstaedt et al.'s (2015a) data files from the relevant Open Science Foundation (OSF) repository, and the majority of the analyses that follow are based on these data" ${ }^{1}$ We did not have access to the original Twitter "Garden Hose" data set, so our analyses rely on the summaries of word usage provided by Eichstaedt et al. in their data files. Next, we downloaded county-aggregated, age-adjusted mortality data for 2009 and 2010 for the ICD-10 category I25.1 (atherosclerotic heart disease) from the Centers for Disease

${ }^{1}$ An earlier version of the present article, available in preprint form at https://psyarxiv.com/dursw, stated that we had not been able to obtain Eichstaedt et al.'s (2015a) code because it had not been made available in the same OSF repository as the data. We are happy to acknowledge here that Eichstaedt and colleagues had in fact made their code available on the website of their Differential Language Analysis ToolKit (DLATK) software project, a fact that they have now documented in their recent preprint (Eichstaedt et al., 2018). We followed the installation instructions for DLATK and were able to reproduce the analyses described by Eichstaedt et al. (2015a, p. 161) under the heading of "Predictive models". 
207 Control and Prevention (CDC) online public health database, known as Wonder

208 (http://wonder.cdc.gov/), in order to check that we could reproduce Eichstaedt et al.'s (2015a)

209 data set exactly. We also downloaded comparable mortality data for the ICD-10 categories X60-

210 X84, collectively labeled "Intentional self-harm" - in order to test the idea that suicide might be

211 at least as well predicted by Twitter language as AHD — as well as the data for several other

212 causes of death (including all-cause mortality) for comparison purposes. Finally, we obtained

213 data about the precise geographical locations of counties from the US Census Bureau

214 (http://www.census.gov/geo/www/gazetteer/files/Gaz_counties_national.zip). All of our

215 statistical analyses were performed in R; a copy of the analysis code can be found at

216 https://osf.io/g42dw.

217

218

219

220

221

222

223

224

225

226

227

228

\section{Results}

Using data downloaded from the CDC Wonder database, we were able to reproduce Eichstaedt et al.'s (2015a) principal data file, named countyoutcomes.csv, exactly. We were also able to reproduce Eichstaedt et al.'s reported numerical results from their article to within rounding error using our own R code (for the dictionary and topic language variables) or by running their modeling software in a virtual machine environment (for the analyses under the heading of "Predictive models").

In the following sections, we report a number of findings that we made when exploring Eichstaedt et al.'s (2015a) data set and the other data that we obtained. The order of these sections follows the same structure that we used in the introduction of the present article, showing how these findings relate to the concerns that we expressed earlier under three broad headings: (a) the use of mortality from AHD as the outcome variable; (b) the use of county-level 
229 aggregated data; and (c) the use of patterns of language in posts to Twitter as the principal

230 predictor of the outcome.

231 Variability in ICD-10 coding of cause of death

232 The validity of the mortality data from the CDC, and in particular the ICD-10 coding of

233 the cause of death, is crucial to establishing the validity of Eichstaedt et al.'s (2015a) findings.

234 Examination of the mortality figures from the counties included in Eichstaedt et al.'s study

235 shows that death rates from AHD in 2009-2010 ranged from 13.4 per 100,000 people (1.55\% of

236 all recorded deaths) in East Baton Rouge Parish, LA to 185.0 per 100,000 people $(26.11 \%$ of all

237 recorded deaths) in Richmond County, NY. It is not clear that any county-level environmental,

238 economic, or social factors that might contribute to the development of AHD would be sufficient

239 to explain why this condition - which, as Eichstaedt et al. noted, is the single most prevalent

240 ICD-10 cause of mortality in the United States - appears to cause 13.8 times more deaths in one

241 county than another. The two counties just mentioned are both among the $10 \%$ most populous

242 counties in the US, with a total of 7,001 and 6,907 deaths, respectively, being recorded there in

243 2009-2010; this suggests that the large difference in recorded mortality from AHD is unlikely to

244 be a statistical fluke due to a limited sample. In contrast, for deaths from cancer, the range of per-

245 county mortality rates across the 1,347 counties included by Eichstaedt et al. runs from 102.4 to

246326.7 per 100,000 people — a factor of 3.19 from lowest to highest, which is little different from

247 the range for all-cause mortality (478.8 to 1390.3 per 100,000 people, a factor of 2.90 from

248 lowest to highest). Eichstaedt et al.'s acknowledgement that "the coding on death certificates

249 may be inconsistent" (p. 166) would thus appear to be somewhat of an understatement, at least as

250 far as AHD is concerned. Indeed, it seems possible that at least part of the variance in AHD

251 mortality (whether this is to be explained by "community-level psychological characteristics" or 
252 some other mechanism) might be due, not to differences in the actual prevalence of AHD as the

253 principal cause of death, but rather to variations in the propensity of local physicians to certify

254 the cause of death as AHD (McAllum, St. George, \& White, 2005; Messite \& Stellman, 1996;

255 Stausberg, Lehmann, Kaczmarek, \& Stein, 2006). Researchers who intend to study AHD

256 mortality using county-level data may wish to take this possibility into account (cf. Roth et al., 257 2017).

258 Use of mortality from AHD as the outcome variable

259 The chronic nature of AHD implies that, to the extent that its prevalence may be affected

260 by deleterious or protective lifestyle and social factors - including Eichstaedt et al.'s (2015a,

261 p. 164) purported "indicators of community-level psychosocial health" —it will have been

262 necessary for these factors to have exerted their effects over a long period. However, there are

263 two potential sources of discrepancy between the current psychosocial state of a person's county

264 of residence and its past effects on their health. First, the socioeconomic climate of an area can

265 change substantially in less than a generation. The decline in the fortunes of the city of Detroit

266 provides a recent dramatic example of this (LeDuff, 2014), but economic development can also

267 bring rapid positive change to parts of a state within quite a short time. Second, individuals tend

268 not to stay in one place; the county where someone spends his or her childhood may be a long

269 way from where he or she ends up working, and possibly different again from where the same

270 person is living when symptoms of AHD emerge later in life, which might be after retirement.

271 Data from the U.S. Census Bureau (2011) show that in 2010 approximately $3.5 \%$ of Americans

272 moved either to another county in the same state, or to another state altogether, a figure that

273 appears from an examination of comparable data from other years to be relatively constant over

274 time. Thus, it seems likely that a substantial proportion of the people who die from AHD each 
275 year in any given county may have lived in one or more other counties during the decades when

276 AHD was developing, and thus been exposed to different forms, both favorable and unfavorable,

277 of Eichstaedt et al.'s purported community-level psychological characteristics during that period.

278 We suggested earlier that if a county-level psychological factor was sufficiently strong to

279 influence mortality from AHD, it might also be expected to influence local rates of suicide. We

280 therefore examined the relationship of the set of causes of death listed by the CDC as "self-

281 harm" with Twitter language usage, using the procedures reported in the first subsections entitled

282 "Language variables from Twitter" and "Statistical analysis" of Eichstaedt et al.'s (2015a,

283 p. 161) Method section. Because of a limitation of the CDC Wonder database, whereby county-

284 aggregated mortality data are only returned for any given county when at least 10 recorded

285 deaths per year, on average, match the requested criteria for the period in question, data for self-

286 harm were only available for 741 counties; however, these represented $89.9 \%$ of the population

287 of Eichstaedt et al.'s set of 1,347 counties.

288 In the "Dictionaries" analysis, we found that mortality from self-harm was negatively

289 correlated with all five "negative" language factors, with three of these correlations (for anger,

290 negative-relationship, and negative-emotion words) being statistically significant at the .05 level

291 (see our Table 1). That is, counties whose residents made greater use of negative language on

292 Twitter had lower rates of suicide, or, to borrow Eichstaedt et al.'s (2015a, p. 162) words, use of

293 negative language was "significantly protective" against self-harm; this statistical significance

294 was unchanged when income and education were added as covariates. In a further contrast to

295 AHD mortality, two of the three positive language factors (positive relations and positive

296 emotions) were positively correlated with mortality from self-harm, although these correlations

297 were not statistically significant at the conventional .05 level. 
299 the "Topics" level. Among the topics most highly correlated with increased risk of self-harm

300 were those associated with spending time surrounded by nature (e.g., grand, creek, hike;

$\left.301 r=.214, \mathrm{CI}^{2}=[.144, .281]\right)$, romantic love (e.g., beautiful, love, girlfriend; $r=.176$

$302 \mathrm{CI}=[.105, .245])$, and positive evaluation of one's social situation (e.g., family, friends,

303 wonderful; $r=.175, \mathrm{CI}=[.104, .244])$. There were also topics of discussion that appeared to be

304 strongly "protective" against the risk of self-harm, such as baseball (e.g., game, Yankees, win;

$305 r=-.317, \mathrm{CI}=[-.381,-.251])$, binge drinking (e.g., drunk, sober, hungover; $r=-.249$,

$306 \mathrm{CI}=[-.316,-.181])$, and watching reality TV (e.g., Jersey, Shore, episode; $r=-.200$,

$307 \mathrm{CI}=[-.269,-.130])$. All of the correlations between these topics and self-harm outcomes, both

308 positive and negative, were significant at the same Bonferroni-corrected significance level (i.e.,

$309.05 / 2,000=.000025)$ used by Eichstaedt et al. (2015a), and remained significant at that level

310 after adjusting for income and education. That is, several topics that were ostensibly associated

311 with "positive," "eudaimonic" approaches to life predicted higher rates of county-level self-harm

312 mortality, whereas apparently hedonistic topics were associated with lower rates of self-harm

313 mortality, and the magnitude of these associations was at least as great — and in a few cases, even

314 greater - than those found by Eichstaedt et al. These topics are shown in "word cloud" form

315 (generated at https://www.jasondavies.com/wordcloud/) in our Figure 1 (cf. Eichstaedt et al.'s

316 Figure 1).

\footnotetext{
${ }^{2}$ We report 95\% CIs here for consistency with Eichstaedt et al. (2015a); however, given their use of a Bonferroni-corrected significance threshold, it could be argued that Eichstaedt et al. should have reported $99.9975 \%$ CIs.
} 
318 to have shown the existence of "community-level psychological factors that are important for the

319 cardiovascular health of communities." Apparently the "positive" versions of these factors, while

320 acting via some unspecified mechanism to make the community as a whole less susceptible to

321 developing hardening of the arteries, also simultaneously manage to make the inhabitants of

322 those counties more likely to commit suicide, and vice versa. We suggest that more research into

323 the possible risks of increased levels of self-harm might be needed before "community-level

324 psychological factors" were to be made the focus of intervention, as Eichstaedt et al. suggested

325 in the final sentence of their article. ${ }^{3}$

\section{Bias caused by selection of counties}

As noted above, the CDC Wonder database returns county-aggregated mortality data for any given cause of death only for those counties where at least 10 deaths from that cause were recorded per year, on average, during the period covered by the user's request. This cutoff means that Eichstaedt et al.’s (2015a) data set is skewed toward counties having higher rates of AHD.

331 For example, AHD mortality was sufficiently high in Plumas County, CA (2010 population

332 20,007; 25 deaths from AHD in 2009-2010) for this county to be included, whereas the

333 corresponding prevalence in McKinley County, NM (2010 population 71,492; 17 deaths from ${ }^{3}$ In their recent preprint, Eichstaedt et al. (2018) noted that the relation between self-harm and positive Twitter language disappeared when they added measures of county-level rurality and elevation as covariates. We do not find this surprising. Our point is not that Twitter language actually predicts county-level mortality from suicide (or AHD); rather, it is that with a sufficient number of predictors, combined with unreliability in the measurement of these, one can easily find spurious relations between variables (cf. Westfall \& Yarkoni, 2016). 
334 AHD in 2009-2010), as well as 130 other counties with higher populations than Plumas County

335 but fewer than 20 deaths from AHD, was not. Thus, the selection of counties tends to include

336 those with higher levels of the outcome variable, which has the potential to introduce selection

337 bias (Berk, 1983). For example, counties with a 2010 population below the median $(78,265)$ in

338 Eichstaedt et al.'s data set had significantly higher AHD mortality rates than counties with larger

339 populations (53.21 versus 49.87 per 100,$000 ; t(1344.6)=3.12, p=.002, d=0.17)$. The effect of

340 such bias on the rest of Eichstaedt et al.'s analyses is hard to estimate, but given that one of these

341 authors' headline results - namely, that their Twitter-only model predicted AHD mortality

342 "significantly better" than a "traditional" model, a claim deemed sufficiently important to be

343 included in their abstract—had an associated $p$ value of .049, even a very small difference might

344 be enough to tilt this result away from traditional statistical significance.

345 Problems associated with county-level aggregation of data

346 We noted earlier that the diversity among counties made it difficult to imagine that the

347 relation between Twitter language and AHD would be consistent across the entire United States.

348 Indeed, when the counties in Eichstaedt et al.'s (2015a) data set are split into two equal subsets

349 along the median latitude of their centroids (38.6484 degrees North, a line that runs

350 approximately through the middle of the states of Missouri and Kansas), the purported effect of

351 county-level Twitter language on AHD mortality as measured by Eichstaedt et al.'s dictionaries

352 seems to become stronger in the northern half of the US than for the country as a whole, but

353 mostly disappears in the southern half (see our Table 2). There does not appear to be an obvious

354 theoretical explanation for this effect; if anything one might expect the opposite, in view of the

355 observation made previously that counties may play a greater role in people's lives in the South. 
A further issue with Eichstaedt et al.'s (2015a) use of data aggregated at the level of

357

358

359

360

361

362

363

364

365

366

367

368

369

370

371

372

373

374

375

376

377

378

counties is that it resulted in an effective sample size that was much smaller than these authors

suggested. For example, Eichstaedt et al. compared their model to "[t]raditional approaches for collecting psychosocial data from large representative samples ... [which] are based on only [emphasis added] thousands of people" (p. 166). This suggests that these authors believed that their approach, using data that was generated by an unspecified (but implicitly very large) number of different Twitter users, resulted in a more representative data set than one built by examining the behaviors and health status of "only" a few thousand individuals. However, by aggregating their Twitter data at the county level, and merging it with other county-level health and demographic information, Eichstaedt et al. reduced each of their variables of interest to a single number for each county, regardless of that county's population. In effect, Eichstaedt et al.'s data set contains a sample of only 1,347 individual units of analysis, each of which has the same degree of influence on the conclusions of the study. A corollary of this is that, despite the apparently large number of participants overall, a very small group of voluble Twitter users could have a substantial influence in smaller counties. For example, in tweets originating from Jefferson County, WA (population 29,872) just nine instances of the word "fuck" (or derivatives thereof) appear in the entire data set, whereas the same word and its variants appear 9,271 times in the tweets of the residents of Montgomery County, NY (population 50,219), a per-inhabitant rate that is almost 600 times larger. Perhaps community standards of polite discourse vary rather more widely across rural areas of the United States than most people might imagine, but it seems at least equally likely that just a few angry people in mid-state New York are responsible for this avalanche of social media profanity, and that their input may consequently have had rather more impact on Eichstaedt et al.'s results than if those same few individuals had been living in Los 
379 Angeles County, CA (population 9,818,605). This aggregation into counties calls into question

380 Eichstaedt et al.'s claim (p. 166) that an analysis of Twitter language can "generate estimates

381 based on 10s of millions of people"; indeed, it could be that their results are being driven by just

382 a few hundred particularly active Twitter users, particularly those living in smaller counties ${ }^{4}$.

383 Apparent censorship of the Twitter data

384 An examination of the words and phrases that appear in Eichstaedt et al.'s (2015a)

385 dictionaries suggests that some form of censorship may have been applied to the Twitter data.

386 For example, the list of frequencies of each word in the dictionary contains entries for the words

387 "nigga," "niggas," and "niggaz" (which, between them, appeared 1,391,815 times in the

388 analyzed tweets, and at least once in all but five of the 1,347 included counties), but not "nigger"

389 or "niggers." It seems highly unlikely that the most common spelling ${ }^{5}$ of this word would not

390 appear even once in any of the 148 million tweets that were included in Eichstaedt et al.'s

391 analyses. A number of other common epithets for ethnic and religious groups are also absent

392 from the dictionary-frequency table — although a search for such words on Twitter suggests that

393 they are in relatively common usage — as are the words "Jew[s]" and "Muslim[s]." In Appendix

394 B of their recent preprint, Eichstaedt et al. (2018) have indicated that they were previously

395 unaware of this issue, which we therefore presume reflects a decision by Twitter to bowdlerize

396 the "Garden Hose" dataset. Such a decision clearly has substantial consequences for any attempt

${ }^{4}$ Examination of Eichstaedt et al.'s (2015a) data set shows that for 267 counties (19.8\%), less than 100,000 words were included in the database, which corresponds to around 5,000 tweets. However, no indication is available of the number of unique Twitter users per county.

5 The spelling "nigga" is often used by African Americans in a neutral or positive sense; the form "nigger" is the one typically used by members of other groups as a racial slur (Goudet, 2013). 
397 to infer a relation between the use of hostile language on Twitter and health outcomes, which

398 requires that the tweets being analyzed are truly representative of the language being used.

399 Indeed, it could be argued that there are few better examples of language that expresses

400 interpersonal hostility than invective directed towards ethnic or religious groups ${ }^{6}$.

401 Potential sources of bias in the "Topics" database

402

A further potential source of bias in Eichstaedt et al.'s (2015a) analyses, which these

403

authors did not mention in their article or their supplemental documents (Eichstaedt et al., 2015b, 2015c), is that their "Topics" database was derived from posts on Facebook (i.e., not Twitter) by a completely different sample of users, as can be seen at the site from which we downloaded this database (http://wwbp.org/data.html). Furthermore, some of the topics that were highlighted by

407 Eichstaedt et al. in the word clouds ${ }^{7}$ in their Figure 1 contain words that appear to directly contradict the topic label (e.g., "famous" and "lovers" in "Hate, Interpersonal Tension," left panel; "enemy" in "Skilled Occupations," middle panel; "painful" in "Positive Experiences," left panel). There are also many incorrectly spelled words, as in topic \#135 ("can't, wait, afford,

411 move, belive [sic], concentrate"), topic \#215 ("wait, can't, till, tomorrow, meet, tomarrow

412 [sic]"), topic \#467 (“who's, guess, coming, thumbs, guy, whos [sic], idea, boss, pointing”), and

413 many topics make little sense at all, such as \#824 (“tooo, sooo, soooo, alll, sooooo, toooo, goood,

\footnotetext{
${ }^{6}$ We assume that the apparent omission of "Jew[s]" and "Muslim[s]" was motivated by concerns that at least some of the tweets mentioning these words might be expressing hostility towards these groups.

${ }^{7}$ It appears that the relative size of the words in Eichstaedt et al.'s (2015a, Figure 1) word clouds is determined by the relative frequency of these words in the Facebook data from which the topics were derived, and does not represent the prevalence of these words in the Twitter data.
} 
414 meee, meeee, youuu, gooo, soooooo, allll, gooood, ohhh, ughh, ohhhh, goooood, mee,

415 sooooooo") and \#983 (“ur, urself, u'll, coz, u've, cos, urs, bcoz, wht, givin”). The extent to which

416 these automatically extracted topics from Facebook really represent coherent psychological or

417 social themes that might appear with any frequency in discussions on Twitter seems to be

418 questionable, in view of the different demographics and writing styles on these two networks.

\section{Flexibility in interpretation of dictionary data}

A problem for Eichstaedt et al.'s (2015a) general argument about the salutary effects of

421 "positive" language was the fact that the use of words expressing positive relationships appeared,

422 in these authors' initial analyses, to be positively correlated with AHD mortality. To address this,

423 Eichstaedt et al. took the decision to eliminate the word love from their dictionary of positive-

424 relationship words. Their justification for this was that "[r]eading through a random sample of

425 tweets containing love revealed them to be mostly statements about loving things, not people"

426 (p. 165). However, similar reasoning could be applied to many other dictionary words-

427 including those that featured in results that did not contradict Eichstaedt et al.'s hypotheses-

428 with the most notable among these being, naturally, hate. In fact, it turns out that hate dominated

429 Eichstaedt et al.'s negative relationships dictionary (41.6\% of all word occurrences) to an even

430 greater degree than love did for the positive relationships dictionary $(35.8 \%)$. We therefore

431 created an alternative version of the negative relationships dictionary, omitting the word hate,

432 and found that, compared to the original, this version was far less likely to produce a statistically

433 significant regression model when predicting AHD mortality (e.g., regressing AHD on negative

434 relationships, controlling for income and education: with hate included, partial $r=.107, p<.001$,

$43595 \% \mathrm{CI}=[.054, .159]$; with hate excluded, partial $r=-.005, p=.849,95 \% \mathrm{CI}=[-.057, .048])$.

436 However, we do not have access to the full Twitter "Garden Hose" data set, and are thus unable 
437 to establish whether, analogously to what Eichstaedt et al. did for love, a similar examination of a

438 "random sample of tweets" containing the word hate might "reveal" that they are mostly

439 statements about hating things rather than people, thus providing an equivalent justification for

440 dropping this word from the analyses.

441 In their Note 6, Eichstaedt et al. (2015a, p. 167) mentioned another justification for

442 removing the word love from the positive relationships dictionary. They noted that "in lower-

443 SES areas, users [may] share more about personal relationships on Twitter, which distorts the

444 results obtained when using the original positive-relationships dictionary." Of course, it might be

445 true of personal relationships, or indeed any other aspect of people's lives, that those who live in

446 lower-SES areas — or, for that matter, those who are married, or smoke, or suffer from diabetes-

447 tend to communicate more (or, indeed, less) about that topic on Twitter. But the factor analysis

448 in Eichstaedt et al.'s Note 6 does not provide any direct evidence for their claim of a possible

449 relation between residence in a lower SES area and a tendency to tweet about personal

450 relationships ${ }^{8}$.

451 Comparison of Twitter-based and "traditional" prediction models

452 Eichstaedt et al. (2015a, p. 161) reported that they "created a single model in which all of

453 the word, phrase, dictionary, and topic frequencies were independent variables and the AHD

454 mortality rate was the dependent variable." It is not clear exactly how this model was constructed

455 or what weighting was given to the various components, even though the numbers of each

456 category (words, phrases, dictionary entries, and topics) vary widely. In their Results section,

\footnotetext{
${ }^{8}$ Eichstaedt et al. (2018) have recently explained that the exclusion of "love" from their positive relationships dictionary in their earlier article (Eichstaedt et al., 2015a) was the result of discussions with a reviewer of.that article.
} 
457 under the heading "Predictions," these authors compared the performance of what we might call

458 their "Twitter omnibus" model with what they described as "traditional" models (i.e., those

459 based on their demographic, socioeconomic, and health status variables), and claimed that the

460 performance of the Twitter-based model was "significantly" better, based on $p$ values of .026 and

461 .049. However, the degrees of freedom here $(1,346)$ are sufficiently numerous, and thus the

462 statistical power to detect an effect sufficiently high, that these $p$ values arguably constitute quite

463 strong evidence in favor of the null hypothesis of no effect (cf. Lakens \& Evers, 2014). It is also

464 unclear from Eichstaedt et al.'s (2015b) Supplemental Material exactly how many predictors

465 were finally included in their model.

466 How similar are the comparative maps?

467 Figure 3 of Eichstaedt et al.'s (2015a) article shows two maps of the counties of the

468 northeastern United States, with those counties that had sufficient cases of AHD mortality to be

469 included in their sample being color-coded according to either CDC-reported or Twitter-

470 predicted AHD mortality. Eichstaedt et al. (2015a, p. 164) claimed that "a high degree of

471 agreement is evident" between the two maps. We set out to evaluate this claim by examining the

472 color assigned to each county on each of the two maps, and determining the degree of difference

473 between the mortality rates corresponding to those colors. To this end, we wrote a program to

474 extract the colors of each pixel across the two maps, convert these colors to the corresponding

475 range of AHD mortality rates, and draw a new map that highlights the differences between these

476 rates using a simple color scheme. The color-based scale shown at the bottom of Eichstaedt et

477 al.'s Figure 3 seems to imply that the maps are composed of 10 different color codes, each

478 representing a decile of per-county AHD mortality, but in fact this scale is somewhat misleading.

479 In fact, 14 different colors (plus white) are used for the counties in the maps in Eichstaedt et al.'s 
480 Figure 3, with each color apparently (assuming that each color corresponds to an equally-sized

481 interval) representing around 7 percentile rank places, or what we might call a "quattuordecile,"

482 of the AHD mortality distribution. For more than half (323 out of 608 , or $53.1 \%$ ) of the counties

483 that have a color other than white in Eichstaedt et al.'s maps, the difference between the two

484 maps is three or more of these 7-point "color intervals," as shown in our Figure 2. Within these

485 counties, the mean discrepancy is at least 29.5 percentage points, and probably considerably

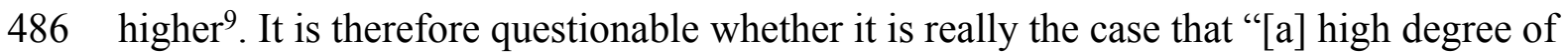

487 agreement is evident" (Eichstaedt et al., 2015, p. 164) between the two maps, such that one

488 might use the Twitter-derived value to predict AHD mortality for any practical purpose.

\section{$\underline{\text { Discussion }}$}

In the preceding paragraphs, we have examined a number of aspects of Eichstaedt et al.'s

491 (2015a) claims about the county-level effects of Twitter language on AHD mortality, using for

492 the most part these authors' own data. We have shown that many of these claims are, at the least, open to alternative interpretations. The coding of their outcome variable (mortality from AHD) is

${ }^{9}$ More precisely, 200 counties (32.9\%) had a discrepancy of three or more color intervals, while 123 counties $(20.2 \%)$ had a discrepancy of six or more. Assuming for simplicity that rounding error is uniformly distributed, a difference of three intervals corresponds to a mean difference of 21.4 percentage points, and a difference of six intervals to a mean difference of 42.8 percentage points. Thus, even with the extremely conservative simplifying assumption that "three (six) or more color intervals" actually means "exactly three (six) intervals," the mean discrepancy across these counties is $((200 \times 21.4)+(123 \times 42.8)) / 323=29.5$ percentage points. Note also that the possible extent of the discrepancy is bounded at between 7 and 13 color intervals, depending on the relative positions of the two counties along the 1-14 scale. 
494 subject to very substantial variability; the process that selects counties for inclusion is biased; the 495 same regression and correlation models "predict" suicide at least as well as AHD mortality but 496 with almost opposite results (in terms of the valence of language predicting positive or negative 497 outcomes) to those found by Eichstaedt et al.; the Twitter-based dictionaries appear not to be a 498 faithful summary of the words that were actually typed by users; arbitrary choices were 499 apparently made in some of the dictionary-based analyses; the topics database was derived from 500 a completely different sample of participants who were using Facebook, not Twitter; there are 501 numerous problems associated with the use of counties as the unit of analysis; and the predictive 502 power of the model, including the associated maps, appears to be questionable. While we were 503 able to reproduce — at a purely computational level — the results of Eichstaedt et al.'s advanced 504 prediction model, based on ridge regression and $k$-fold cross-validation, we do not believe that 505 this model can address the problems of validity and reliability posed by the majority of the points 506 just mentioned. In summary, the evidence for the existence of community-level psychological 507 factors that determine AHD mortality better than traditional socioeconomic and demographic 508 predictors seems to be considerably less strong than Eichstaedt et al. claimed.

509 A tourist, or indeed a field anthropologist, driving through Jackson County (2010

510 population 42,376) and Clay County (2010 population 26,890) in southern Indiana might not

511 notice very much difference between the two. According to the US Census Bureau

512 (https://factfinder.census.gov/) these counties have comparable population densities, ethnic

513 make-ups, and median household incomes. It seems unlikely that there would be a large variation

514 in the "norms, social connectedness, perceived safety, and environmental stress, that contribute

515 to health and disease" (Eichstaedt et al., 2015a, pp. 159-160) between these two rural

516 Midwestern communities. Yet, according to Eichstaedt et al.'s data, the levels of anger and 
517 anxiety expressed on Twitter were more than 12 and 6 times, respectively, higher in Jackson

518 County than in Clay County. These differences are not easy to explain; any community-level

519 psychological characteristics that might be driving them must obey some strange properties.

520 Such characteristics would have to operate, at least partly, in ways that are not accounted for by

521 variables for which Eichstaedt et al. applied statistical controls (such as income and smoking

522 prevalence), yet presumably they must have some physical manifestation in order to be able to

523 have an effect on people's expressed feelings. It is difficult to imagine how such a characteristic

524 might have gone unnoticed until now, yet be able to cause people living in very similar

525 socioeconomic conditions less than 100 miles apart in the same state to express such varying

526 degrees of negative emotionality. A more parsimonious explanation, perhaps, is that there is a

527 very large amount of noise in the measures of the meaning of Twitter data used by Eichstaedt et

528 al., and these authors' complex analysis techniques (involving, for example, several steps to deal

529 with high multicollinearity) are merely modeling this noise to produce the illusion of a

530 psychological mechanism that acts at the level of people's county of residence. Certainly, the

531 different levels of "negative" Twitter language between these two Indiana counties appear to

532 have had no deleterious differential effect on local AHD mortality; indeed, at 45.4 deaths per

533 100,000 inhabitants, “angry” Jackson County's AHD mortality rate in 2009-2010 was 23.7\%

534 lower than "laid-back" Clay County's (59.5 per 100,000 inhabitants). As we showed in our

535 analysis of Eichstaedt et al.'s comparative maps, this failure of Twitter language to predict AHD

536 mortality with any reliability is widespread.

537 In a recent critique, Jensen (2017) examined the claims made by Mitchell, Frank, Harris,

538 Dodds, and Danforth (2013) regarding the ability of Twitter data to predict happiness. Jensen

539 argued that "the extent of overlap between individuals' online and offline behavior and 
540 psychology has not been well established, but there is certainly reason to suspect that a gap exists

541 between reported and actual behavior" (p. 2). Jensen went on to raise a number of other points

542 about the use of Twitter's "garden hose" dataset that appear to be equally applicable to

543 Eichstaedt et al. (2015a), concluding that "When researchers approach a data set, they need to

544 understand and publicly account for not only the limits of the data set, but also the limits of

545 which questions they can ask ... and what interpretations are appropriate" (p. 6). It is worth

546 noting that Mitchell et al. were attempting to predict happiness only among the people who were

547 actually sending the tweets that they analyzed. While certainly not a trivial undertaking, this

548 ought to be considerably less complex than Eichstaedt et al.'s attempt to predict the health of one

549 part of the population from the tweets of an entirely separate part (cf. their comment on p. 166:

550 “The people tweeting are not the people dying"). Hence, it would appear likely that Jensen's

551 conclusions - namely, that the limitations of secondary data analyses and the inherent noisiness

552 of Twitter data meant that Mitchell et al.'s claims about their ability to predict happiness from

553 tweets were not reliably supported by the evidence-would be even more applicable to

554 Eichstaedt et al.'s study, unless these authors could show that they took steps to avoid the

555 deficiencies of Mitchell et al. On a related theme, Robinson-Garcia, Costas, Isett, Melkers, and

556 Hicks (2017) warned that bots, or humans tweeting like bots, represent a considerable challenge

557 to the interpretability of Twitter data; this theme has become particularly salient in view of recent

558 claims that a substantial proportion of the content on Twitter and other social media platforms

559 may not represent the spontaneous output of independent humans (Varol, Ferrara, Davis,

560 Menczer, \& Flammini, 2017).

561 The principal theoretical claim of Eichstaedt et al.'s (2015a) article appears to be that the

562 best explanation for the associations that were observed between county-level Twitter language 
563 and AHD mortality is some geographically-localized psychological factor, shared by the

564 inhabitants of an area, that exerts ${ }^{10}$ a substantial influence on aspects of human life as different as

565 vocabulary choice on social media and arterial plaque accumulation, independently of other

566 socioeconomic and demographic factors. However, Eichstaedt et al. did not provide any direct

567 evidence for the existence of these purported community-level psychological characteristics, nor

568 of how they might operate. Indeed, we have shown that the same techniques that predicted AHD

569 mortality could equally well have been used to predict county-level suicide prevalence, with the

570 difference that higher rates of self-harm seem to be associated with "positive" Twitter language.

571 Of course, there is no suggestion that the study of the language used on Twitter by the

572 inhabitants of any particular county has any real predictive value for the local suicide rate; we

573 believe that such associations are likely to be the entirely spurious results of imperfect

574 measurements and chance factors, and to use Twitter data to predict which areas might be about

575 to experience higher suicide rates is likely to prove extremely inaccurate (and perhaps ethically

${ }^{10}$ Eichstaedt et al. (2015a) included a disclaimer about causality on p. 166 of their article.

However, we feel that this did not adequately compensate for some of their language elsewhere in the article, such as "Local communities create [emphasis added] physical and social environments that influence [emphasis added] the behaviors, stress experiences, and health of their residents" (p. 166; both of the italicized words here seem to us to imply causation at least as strongly as our word "exerts"), and "Our approach ... could bring researchers closer to understanding the community-level psychological factors that are important for the cardiovascular health of communities and should become the focus of intervention" (p. 166, seemingly implying that an intervention to change these psychological factors would be expected to lead to a change in cardiovascular health). 
576 questionable as well). We believe that it is up to Eichstaedt et al. to show convincingly why these

577 same considerations do not apply to their analyses of AHD mortality; as it stands, their article

578 does not do this. Taken in conjunction with the pitfalls (Westfall \& Yarkoni, 2016) of including

579 imperfectly-measured covariates (such as Eichstaedt et al.'s county-level measures of smoking

580 and health status, as described above) in regression models, and the likely presence of numerous

581 substantial but meaningless correlations in any data set of this type (the "crud factor"; Meehl,

$5821990^{11}$ ), it seems entirely possible that Eichstaedt et al.'s conclusions might be no more than the

583 result of fitting a model to noise.

584

585

586

587

588

589

590

591

592

593

594

\section{Conclusions}

It appears that the existence of community-level psychological characteristics - and their presumed valence, either being "protective" or "risk" factors for AHD mortality—was inferred by Eichstaedt et al. (2015a) from the rejection of a series of statistical null hypotheses which, though not explicitly formulated by these authors, appear to be of the form "There is no association between the use of [a 'positive' or 'negative' language element] by the Twitter users who live in a given county, and AHD-related mortality among the general population of that county." Yet, the rejection of a statistical null hypothesis cannot in itself justify the acceptance of any particular alternative hypothesis (Dienes, 2008) —especially one as vaguely specified as the existence of Eichstaedt et al.'s purported county-level psychological characteristics that operate via some unspecified mechanism — in the absence of any coherent theoretical explanation.

${ }^{11}$ For example, using data from the CDC for the 2009-2010 period, county-level mortality from assault is strongly correlated with county-level mortality from cancer $(r=.55)$, but completely uncorrelated with county-level mortality from AHD $(r=.00)$. There seems to be no obvious theoretical explanation for these results. 
595 Indeed, it seems to us that Eichstaedt et al.'s results could probably equally well be used to

596 justify the claim that the relation between Twitter language and AHD mortality is being driven

597 by county-level variations in almost any phenomenon imaginable. To introduce a new

598 psychological construct without a clear definition, and whose very existence has only been

599 inferred from a correlational study—as Eichstaedt et al. did—is a very risky undertaking indeed. 


\section{References}

602 Abrams, S. J., \& Fiorina, M. P. (2012). “The Big Sort” that wasn't: A skeptical reexamination.

603 PS: Political Science \& Politics, 45, 203-210.

604 http://dx.doi.org/10.1017/S1049096512000017

605

606

607

608

609

610

611

612

613

614

615

616

617

618

619

620
Association for Psychological Science. (2015). Language on Twitter tracks rates of coronary heart disease. Retrieved from http://www.psychologicalscience.org/news/releases/twitter-usage-can-predict-rates-ofcoronary-heart-disease.html

Benton, J. E. (2002). Counties as service delivery agents: Changing expectations and roles. Westport, CT: Praeger.

Berk, R. A. (1983). An introduction to sample selection bias in sociological data. American Sociological Review, 48, 386-398.

Beyer, K. M. M., Schultz, A. F., \& Rushton, G. (2008). Using ZIP® codes as geocodes in cancer research. In G. Rushton, M. P. Armstrong, J. Gittler, B. R. Greene, C. E. Pavlik, M. M. West, \& D. L. Zimmerman (Eds.), Geocoding health data: The use of geographic codes in cancer prevention and control, research, and practice (pp. 37-67). Boca Raton, FL: CRC Press.

Clark, A. M., DesMeules, M., Luo, W., Duncan, A. S., \& Wielgosz, A. (2009). Socioeconomic status and cardiovascular disease: Risks and implications for care. Nature Reviews Cardiology, 6, 712-722. http://dx.doi.org/10.1038/nrcardio.2009.163 
621 Eichstaedt, J. C., Schwartz, H. A., Kern, M. L., Park, G., Labarthe, D. R., Merchant, R. M., .. .

622 Seligman, M. E. P. (2015a). Psychological language on Twitter predicts county-level

623 heart disease mortality. Psychological Science, 26, 159-169.

624 http://dx.doi.org/10.1177/0956797614557867

625 Eichstaedt, J. C., Schwartz, H. A., Kern, M. L., Park, G., Labarthe, D. R., Merchant, R. M., . .

626 Seligman, M. E. P. (2015b). Supplemental Method. Retrieved from

627 http://journals.sagepub.com/doi/suppl/10.1177/0956797614557867

628

629

Eichstaedt, J. C., Schwartz, H. A., Kern, M. L., Park, G., Labarthe, D. R., Merchant, R. M., .. . Seligman, M. E. P. (2015c). Supplemental Tables. Retrieved from

630 http://wwbp.org/papers/PsychSci2015_HeartDisease_Tables.pdf

631

632

633

634

635

636

637

638

639

640

641

642

643

Eichstaedt, J. C., Schwartz, H. A., Giorgi, S., Kern, M. L., Park, G., Sap, M., . . Ungar, L. H. (2018). More evidence that Twitter language predicts heart disease: A response and replication. Retrieved from https://psyarxiv.com/p75ku/

Franklin, J. C., Ribeiro, J. D., Fox, K. R., Bentley, K. H., Kleiman, E. M., Huang, X., . . Nock, M. K. (2017). Risk factors for suicidal thoughts and behaviors: A meta-analysis of 50 years of research. Psychological Bulletin, 143, 187-232. http://dx.doi.org/10.1037/bul0000084.

Friedman, M., \& Rosenman, R. (1959). Association of specific overt behaviour pattern with blood and cardiovascular findings. Journal of the American Medical Association, 169, 1286-1296. http://dx.doi.org/10.1001/jama.1959.03000290012005

Funk, S. D., Yurdagul, A., Jr., \& Orr, A. W. (2012). Hyperglycemia and endothelial dysfunction in atherosclerosis: Lessons from type 1 diabetes. International Journal of Vascular Medicine, 2012, 569654. http://dx.doi.org/10.1155/2012/569654 
644 Goudet, L. (2013). Alternative spelling and censorship: The treatment of profanities in virtual

645

646

647

648

649

650

651

652

653

654

655

656

657

658

659

660

661

662

663

664 communities. In D. Jamet \& M. Jobert (Eds.), Aspects of linguistic impoliteness (pp. 209222). Cambridge, England: Cambridge Scholars

Haider-Merkel, D. P. (2009). Political encyclopedia of U.S. states and regions. New York, NY: CQ Press.

Hoyert, D. L. (2011). The changing profile of autopsied deaths in the United States, 1972-2007 (NCHS data brief no. 67). Hyattsville, MD: National Center for Health Statistics. Retrieved from https://www.cdc.gov/nchs/data/databriefs/db67.pdf

Izadi, E. (2015, January 21). Tweets can better predict heart disease rates than income, smoking and diabetes, study finds. The Washington Post. Retrieved from https://www.washingtonpost.com/news/to-your-health/wp/2015/01/21/tweets-can-betterpredict-heart-disease-rates-than-income-smoking-and-diabetes-study-finds/

Jacobs, T. (2015, January 21). Happier tweets, healthier communities. Pacific Standard. Retrieved from https://psmag.com/environment/happier-tweets-healthier-communities98710

Jensen, E. A. (2017). Putting the methodological brakes on claims to measure national happiness through Twitter: Methodological limitations in social media analytics. PLoS ONE, 12(9), e0180080. http://dx.doi.org/10.1371/journal.pone.0180080

Kilgore, E. (2012, March 20). Counties as communities. Washington Monthly. Retrieved from http:/www.washingtonmonthly.com/political-animala/2012_03/counties_as_communities036175.php 
665 Kuper, H., Marmot, M., \& Hemingway, H. (2002). Systematic review of prospective cohort 666 studies of psychosocial factors in the aetiology and prognosis of coronary heart disease.

667 Seminars in Vascular Medicine, 2, 267-314. http://dx.doi.org/10.1055/s-2002-35401

668

669

670

671

672

673

674

675

676

677

678

679

680

681

682

683

684

685

686

Practical recommendations to increase the informational value of studies. Perspectives on Psychological Science, 9, 278-292. http://dx.doi.org/10.1177/1745691614528520

Lazer, D., Kennedy, R., King, G., \& Vespignani, A. (2014). The parable of Google Flu: Traps in big data analysis. Science, 343, 1203-1205. http://dx.doi.org/10.1126/science.1248506

LeDuff, C. (2014). Detroit: An American autopsy. New York, NY: Penguin.

Leyland, A. H. (2005). Socioeconomic gradients in the prevalence of cardiovascular disease in Scotland: The roles of composition and context. Journal of Epidemiology \& Community Health, 59, 799-803. http://dx.doi.org/10.1136/jech.2005.034017

Mant, J., Wilson, S., Parry, J., Bridge, P., Wilson, R., Murdoch, W., .. . Warfield, A. (2006). Clinicians didn't reliably distinguish between different causes of cardiac death using case histories. Journal of Clinical Epidemiology, 59, 862-867. http:/dx.doi.org/10.1016/j.jclinepi.2005.11.021

McAllum, C., St. George, I., \& White, G. (2005). Death certification and doctors' dilemmas: A qualitative study of GPs' perspectives. The British Journal of General Practice, 55, 677683.

Meehl, P. E. (1990). Why summaries of research on psychological theories are often uninterpretable. Psychological Reports, 66, 195-244. http://dx.doi.org/10.2466/pr0.1990.66.1.195 
687 Messite, J., \& Stellman, S. D. (1996). Accuracy of death certificate completion: The need for 688 formalized physician training. Journal of the American Medical Association, 275, 794689 796. http://dx.doi.org/10.1001/jama.1996.03530340058030

690 Mitchell, L., Frank, M. R., Harris, K. D., Dodds, P. S., \& Danforth, C. M, (2013). The geography 691 of happiness: Connecting Twitter sentiment and expression, demographics, and objective $692 \quad$ characteristics of place. PLoS ONE, 8(5), e64417. http://dx.doi.org/10.1371/journal.pone.0064417 PMID: 23734200

694 O'Connor, R. C., \& Nock, M. K. (2014). The psychology of suicidal behaviour. The Lancet 695 Psychiatry, 1, 73-85. http://dx.doi.org/10.1016/S2215-0366(14)70222-6 906-912. http://dx.doi.org/10.1007/s00127-004-0873-y

Riggio, R. E. (2014, August 14). Are You a Type A, B, or D Personality? Psychology Today. Retrieved from https://www.psychologytoday.com/blog/cutting-edgeleadership/201408/are-you-type-b-or-d-personality

Riva, M., Gauvin, L., \& Barnett, T. A. (2007). Toward the next generation of research into small 703 area effects on health: A synthesis of multilevel investigations published since July 1998. Journal of Epidemiology \& Community Health, 61, 853-861. http://dx.doi.org/10.1136/jech.2006.050740

Robinson-Garcia, N., Costas, R., Isett, K., Melkers, J., \& Hicks, D. (2017). The unbearable emptiness of tweeting — about journal articles. PLOS ONE, 12(8), e0183551. http://dx.doi.org/10.1371/journal.pone.0183551 
709 Roth, G. A., Dwyer-Lindgren, L., Bertozzi-Villa, A., Stubbs, R. W., Morozoff, C., Naghavi, M.,

710 ... Murray, C. J. L. (2017). Trends and patterns of geographic variation in cardiovascular

711 mortality among US counties, 1980-2014. JAMA, 317, 1976-1992.

712 http://dx.doi.org/10.1001/jama.2017.4150

713 Singal, J. (2015, January 22). Can angry tweets predict heart-disease rates? New York Magazine.

714 Retrieved from http://nymag.com/scienceofus/2015/01/can-angry-tweets-predict-heart-

$715 \quad$ disease-rates.html

716 Smith, T. W. (2006). Personality as risk and resilience in physical health. Current Directions in

717 Psychological Science, 15, 227-231. http://dx.doi.org/10.1111/j.1467-8721.2006.00441.x

718 Smith, T. W. (2011). Toward a more systematic, cumulative, and applicable science of

719 personality and health: lessons from type D personality. Psychosomatic Medicine, 73,

528-532. http://dx.doi.org/10.1097/PSY.0b013e31822e095e

721 Stausberg, J., Lehmann, N., Kaczmarek, D., \& Stein, M. (2006). Reliability of diagnoses coding 722 with ICD-10. International Journal of Medical Informatics, 75, 50-57.

723 http://dx.doi.org/10.1016/j.ijmedinf.2006.11.005

724 Subramanian, S. V., Jones, K., Kaddour, A., \& Krieger, N. (2008). Revisiting Robinson: The 725 perils of individualistic and ecologic fallacy. International Journal of Epidemiology, 38, 342-360. http://dx.doi.org/10.1093/ije/dyn359

727 U.S. Census Bureau. (2011, May 23). Geographical Mobility: 2009 to 2010. Retrieved from 728 https:/www.census.gov/data/tables/2010/demo/geographic-mobility/cps-2010.html 
729 Varol, O., Ferrara, E., Davis, C. A., Menczer, F., \& Flammini, A. (2017, May). Online human-

730 bot interactions: Detection, estimation, and characterization. Paper presented at the 11th

731 International AAAI Conference on Web and Social Media, Montréal, Canada. Retrieved

732 from https://arxiv.org/abs/1703.03107

733 Welsh, J., Korda, R. J., Joshy, G., Butterworth, P., Brown, A., \& Banks, E. (2017). Psychological

734 distress and ischaemic heart disease: cause or consequence? Evidence from a large

735 prospective cohort study. Journal of Epidemiological and Community Health. Advance

736 online publication. http://dx.doi.org/10.1136/jech-2017-209535

737 Westfall, J., \& Yarkoni, T. (2016). Statistically controlling for confounding constructs is harder

738 than you think. PLoS ONE, 11, e0152719.

$739 \quad$ http://dx.doi.org/10.1371/journal.pone.0152719

740 Wilson, S. (2009). Type A and B personalities. The Guardian, March 7. Retrieved from https://www.theguardian.com/lifeandstyle/2009/mar/07/personality-type-a-type-b 


\section{Figure 1}

Twitter Topics Highly Correlated With Age-Adjusted Mortality from Self-Harm, cf. Eichstaedt et al.'s (2015a) Figure 1.

(A-C) Topics positively correlated with county-level self-harm mortality: (A) Friends and family, $r=.175$. (B) Romantic love, $r=.176$. (C) Time spent with nature, $r=.214$.

(D-F) Topics negatively correlated with county-level self-harm mortality: (D) Watching reality TV, $r=-.200$. (E) Binge drinking, $r=-.249$. (F) Baseball, $r=-.317$.

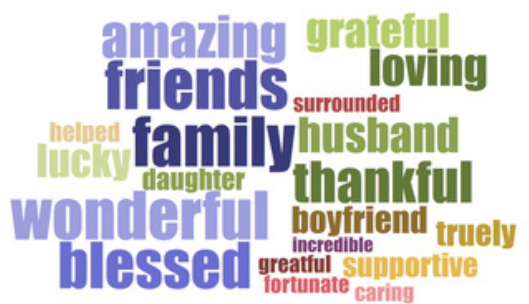

A

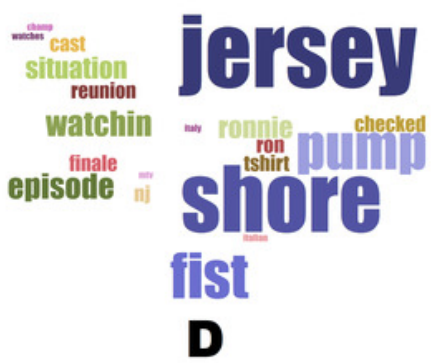

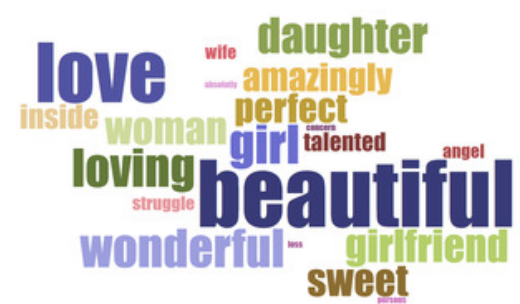

B

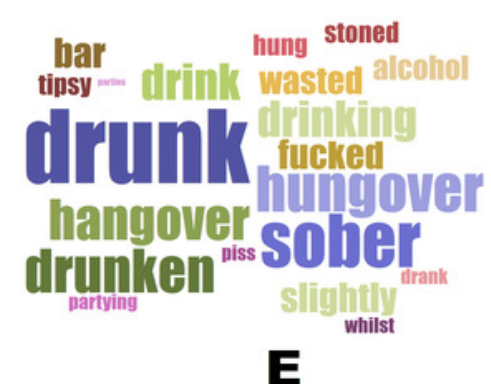

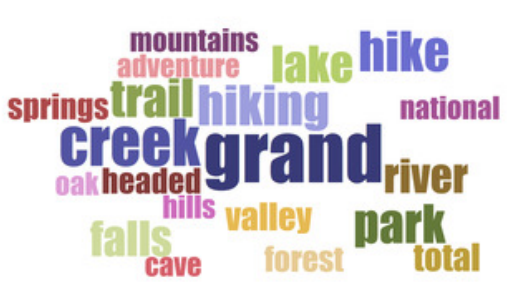

C

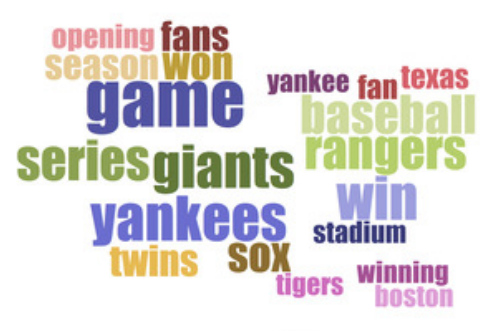

F 
Figure 2

Difference between the two maps of AHD mortality rates (CDC-reported and Twitterpredicted) from Eichstaedt et al.'s (2015a) Figure 3.

Note: Green indicates a difference of $0-2$ color-scale points (see discussion in the text) between the two maps; yellow, a difference of 3-5 points; red, a difference of 6 or more points. Of the 608 colored areas, 123 (20.2\%) are red and 200 (32.9\%) are yellow.

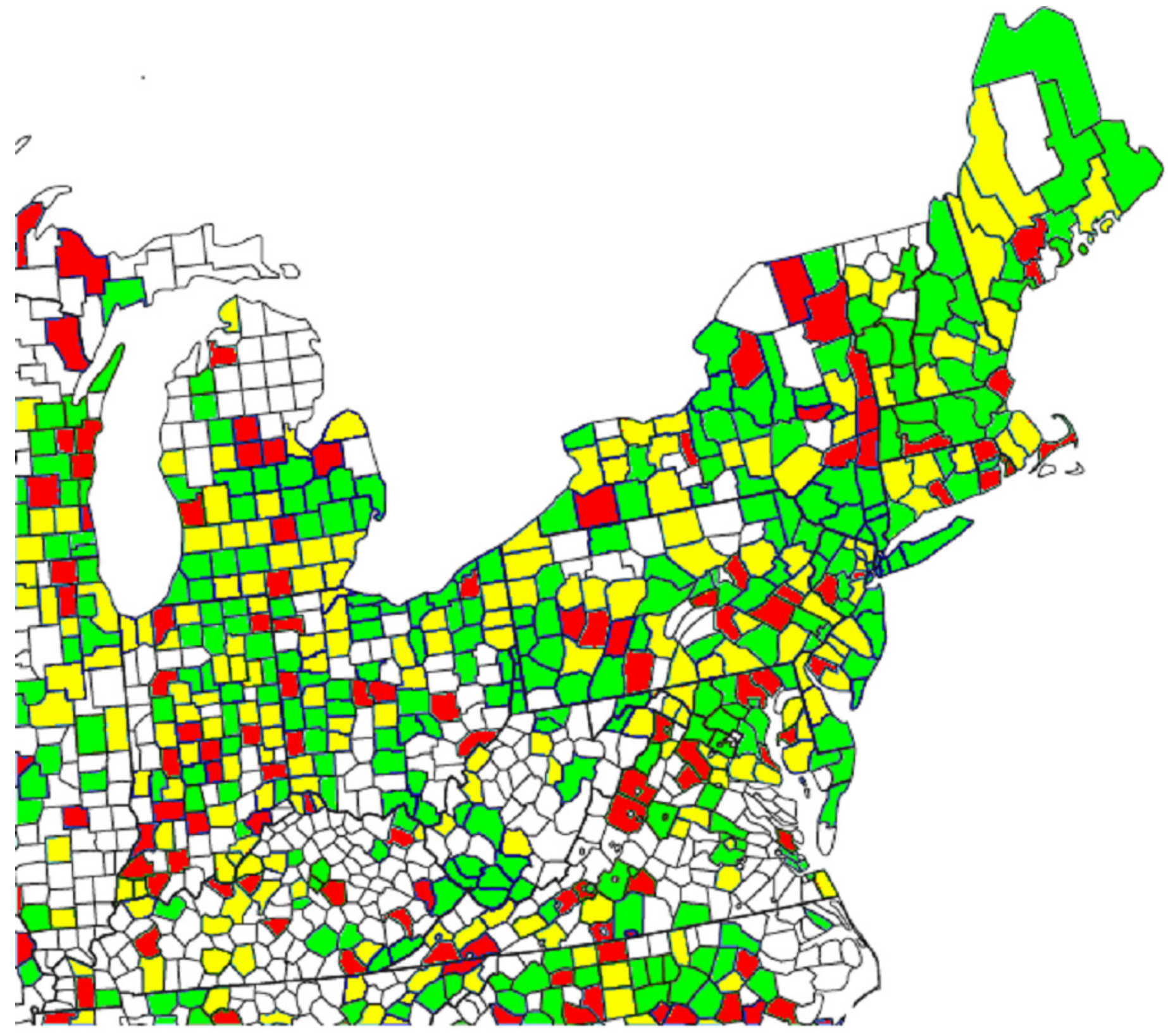




\section{Table $\mathbf{1}$ (on next page)}

Correlations between self-harm and Twitter language measured by dictionaries.

${ }^{a}$ Following Eichstaedt et al. (2015a), the word love was removed from the dictionary for this variable. See discussion in the text. 


\begin{tabular}{lrrr}
\hline Language variable & $r$ & $p$ & $95 \%$ CI \\
\hline Risk factors & & & \\
Anger & -0.169 & $<.001$ & {$[-0.238,-0.099]$} \\
Negative relationships & -0.095 & .010 & {$[-0.166,-0.023]$} \\
Negative emotions & -0.102 & .005 & {$[-0.173,-0.030]$} \\
Disengagement & 0.008 & .831 & {$[-0.064,0.080]$} \\
Anxiety & -0.045 & .219 & {$[-0.117,0.027]$} \\
Protective factors & & & {$[-0.073,0.071]$} \\
Positive relationships ${ }^{\mathrm{a}}$ & -0.001 & .976 & {$[-0.013,0.131]$} \\
Positive emotions & 0.059 & .110 & {$[-0.103,0.041]$} \\
Positive engagement & -0.031 & .393 & \\
\hline
\end{tabular}

1 


\section{Table 2 (on next page)}

Partial correlations between atherosclerotic heart disease (AHD) mortality and Twitter language measured by dictionaries, across the northern and southern halves of the United States.

Partial $r$ : partial correlation coefficients obtained from a regression predicting AHD from the Twitter theme represented by the language variable, with county-level education and income as control variables.

a Following Eichstaedt et al. (2015a), the word love was removed from the dictionary for this variable. See discussion in the text. 


\begin{tabular}{|c|c|c|c|c|c|c|}
\hline \multirow[b]{2}{*}{ Language variable } & \multicolumn{3}{|c|}{ North } & \multicolumn{3}{|c|}{ South } \\
\hline & Partial $r$ & $p$ & $95 \% \mathrm{CI}$ & Partial $r$ & $p$ & $95 \% \mathrm{CI}$ \\
\hline \multicolumn{7}{|l|}{ Risk factors } \\
\hline Anger & 0.240 & $<.001$ & {$[0.168,0.310]$} & -0.020 & .604 & {$[-0.095,0.056]$} \\
\hline Negative relationships & 0.156 & $<.001$ & {$[0.081,0.229]$} & 0.060 & .121 & {$[-0.016,0.135]$} \\
\hline Negative emotions & 0.108 & .005 & {$[0.032,0.182]$} & 0.028 & .462 & {$[-0.047,0.104]$} \\
\hline Disengagement & 0.166 & $<.001$ & {$[0.092,0.239]$} & -0.012 & .750 & {$[-0.088,0.063]$} \\
\hline Anxiety & 0.017 & .654 & {$[-0.058,0.093]$} & 0.104 & .007 & {$[0.028,0.178]$} \\
\hline \multicolumn{7}{|l|}{ Protective factors } \\
\hline Positive relationships ${ }^{\text {a }}$ & -0.032 & .411 & {$[-0.107,0.044]$} & 0.111 & .004 & {$[0.035,0.185]$} \\
\hline Positive emotions & -0.166 & $<.001$ & {$[-0.238,-0.091]$} & 0.082 & .034 & {$[0.006,0.156]$} \\
\hline Positive engagement & -0.192 & $<.001$ & {$[-0.264,-0.119]$} & 0.041 & .288 & {$[-0.035,0.116]$} \\
\hline
\end{tabular}

